\title{
Emergency admission of patients to general surgical beds: attitudes of general practitioners, surgical trainees, and consultants in Liverpool, UK
}

Peter McCulloch, Janet Bowyer, Tim Fitzsimmons, Michael Johnson, Derek Lowe, Richard Ward

\begin{abstract}
Objectives-To determine (a) whether doctors involved in the process of emergency surgical admission could agree about which patients should be admitted, (b) whether there were consistent differences between doctors in different speciality groups, and (c) whether these opinions were greatly influenced by nonclinical factors.

Design-Independent assessment of summarised case histories by three "expert" clinicians (two consultant surgeons and one general practitioner (GP)), by a group of $10 \mathrm{GPs}$, and by a group of 10 junior and senior surgeons. Experts, but not other observers, scored admissions both independently and as a consensus group. Observers indicated for each patient whether they would admit, would not admit, or were unsure.
\end{abstract}

Setting-An urban general hospital with teaching status.

Subjects-Fifty consecutive patients admitted to the general surgical unit as emergencies during 1995.

Main outcome measures-Proportion of admissions considered unnecessary or uncertain: agreement between observers on these proportions: effect of social and procedural factors on the admission decision. Results-Between 8 and 34\% of admissions were considered unnecessary and $20-38 \%$ of unclear necessity. Agreement between the groups of clinicians was not good. GPs and consultant surgeons showed the poorest agreement (kappa $=0.08$ to $0.25,4 \mathrm{com}$ parisons), and the GPs scored a higher percentage of admissions as unnecessary (34 v 8-12\%). After discussion, the consensus group achieved good to very good agreement (kappa 0.61-0.84).

Conclusions-Different groups of doctors vary widely in their views about the need for emergency surgical admission. Good agreement can be reached by consensus discussion. GPs are less likely than surgeons to consider emergency surgical admission necessary.

\section{( $\mathcal{F}$ Epidemiol Community Health 1997;51:315-319)}

The number of emergency admissions to hospitals in the UK has risen by between 40 and $100 \%$ during the past 10 years, with most of the increase being noted since $1991 .{ }^{12}$ The reasons for this increase have been much discussed, but little objective evidence is available. The resulting pressure on beds has had serious effects on the elective work of many units, making this a matter of serious concern for managers, clinicians, and potential patients. Increases in emergency activity imply either a resetting of the threshold for emergency admission or a genuine increase in the burden of disease in the community. The measured changes in the latter resulting from the ageing of the British population and other known demographic and epidemiological changes cannot explain the large and rapid shift in admissions. ${ }^{3}$ Factors that might contribute to the observed changes include new technology leading to routine active treatment of conditions previously not regarded as treatable, social changes leading to loss of home support, loss of previously available alternative care, changes in the organisation and funding of acute surgical services, changes in public attitudes, and changes in medical attitudes.

The last of these is clearly pivotal, since doctors have the responsibility of defining whether acute hospital admissions are necessary or not. We do not know whether there is general agreement within the profession about what constitutes a "necessary" admission, but such a consensus is clearly necessary to allow rational analysis of the problems posed by increasing numbers of such admissions. Surgical emergency admissions have received less attention than medical emergencies, but the rise in numbers appears to affect all specialty groups. We therefore set out to determine the attitudes of the doctors directly involved in admitting surgical patients to hospital. The training and clinical role of different types of doctors may lead to differences of opinion about the appropriate management of patients presenting with acute "surgical" conditions. We therefore asked whether doctors involved in the process of emergency surgical admission could agree about which patients should be admitted, whether there were consistent differences between doctors in different speciality groups, and whether these opinions were greatly influenced by non-clinical factors. To answer these questions we asked doctors involved in the emergency admission process to evaluate case summaries of a series of real emergency admissions to the Fazakerley surgical unit. 


\section{Methods}

OVERALL STUDY PLAN

The purpose of our studies has been to determine the influences that affect the numbers of emergency surgical admissions, in order to decide whether such patients could be managed more efficiently by other means. We have therefore studied (a) clinician estimation of the need for emergency admission, (b) clinical interventions performed or ordered during the first 24 hours of inpatient stay, and (c) social and organisational factors thought likely to have an impact upon the decision to admit. The purpose of studying the social and organisational factors is to form hypotheses about the influences leading to unnecessary inpatient admission. The purpose of studying the clinical interventions was to measure "clinician concern", with the aim of developing a scoring system to substitute for the consensus medical view in future studies. The aim of the current study was to determine the degree of agreement and disagreement on the need for emergency admissions among the members of the profession most closely involved with the process. Issues (b) and (c) noted above will be addressed in future publications.

\section{CURRENT STUDY}

Fifty consecutive emergency admissions to the general surgical service at Fazakerley Hospital during March 1995 were selected as the database for this study. Our general surgical service frequently admits patients who are subsequently referred to allied specialities such as urology or gynaecology, usually because the presenting complaint is one of abdominal pain. Patients admitted directly to specialist surgical disciplines such as maxillo-facial, orthopaedic, or neurosurgery were not included in this study. The presenting complaint, other relevant history, and examination findings were summarised retrospectively in fewer than 500 words by one of the research team, together with the results of immediate investigations normally available to a doctor in the emergency department within two hours of seeing the patient (see example in Appendix I). The summariser (JB) was an experienced nurse who had worked on both $A \& E$ and general surgical units. Summaries were compiled from the emergency department and surgical notes and recorded test results, occasionally supplemented by con-

\section{KEY POINTS}

- General practitioners were much more likely than consultant surgeons to consider an emergency surgical admission to be inappropriate.

- There is a large percentage $(20-38 \%)$ of emergency surgical admissions whose justification is considered uncertain.

- A consensus panel of doctors from different specialties can achieve good agreement on the appropriateness of emergency admission despite their initially differing views. versations with the admitting medical staff. The summariser was responsible for the data collection and compilation, but all summaries were cross checked by a consultant surgeon (PMcC).

The summaries were shown to a group of doctors, who were asked to allocate each case to one of three classes:

- A. Admission definitely required

- B. Unclear on information supplied whether admission warranted or not

- C. Admission definitely not justified.

After making this decision, the doctors were asked to read a separate page outlining for each case some social and organisational factors of possible relevance to the decision to admit. These were as follows:

- Day and time of attendance at the A\&E department;

- Family and social service support available (number and age of household members, their fitness, and any important help from friends, neighbours or relatives, nature and frequency of any current help from social services);

- Suitability of the patients' home for nursing care;

- Availability to the patient of a telephone and/or a car.

The doctors were then asked to review the allocation of the case to A, B, or C. Doctors were not asked to consider how the patient could be best managed or to predicate their decision on the existence of alternatives to hospital admission, but simply to state what they thought they would have done if they were the admitting member of the surgical team. If they felt that a definite decision required additional information that was not supplied in the summary they were asked to score the case as $\mathbf{B}$.

Two consultant surgeons and one GP, who were active participants in the study, each marked all 50 cases. In addition, the cases were divided into groups of five and distributed among $10 \mathrm{GPs}$ selected at random from the audience of a postgraduate meeting at $\mathrm{Fa}$ zakerley Hospital. None of the GPs were trainees: seven were men and three women: their experience in practice ranged from 2-30 years. A group of 10 surgical registrars, senior registrars, and consultants from the directorate of surgery, selected at random from attendees at a surgical audit meeting (attendance at which is compulsory) was also asked to mark five cases each.

After all assessments were made, the study group and the research nurse met to review their evaluation of the cases based on all the information. Where there was disagreement the case summary was discussed, and an attempt made to reach a consensus view. The number of cases allocated to each category, the number in which disagreement remained, and the degrees of agreement between observers were then re-analysed and recorded. 
Table 1 Assessments of emergency admissions: evaluation of clinical data only (evaluation of all data)

\begin{tabular}{lllll}
\hline & $A(+1)$ & $B(0)$ & $C(-1)$ & Score \\
\hline Consultant 1 & $33(34)$ & $14(10)$ & $3(6)$ & $30(30)$ \\
Consultant 2 & $26(29)$ & $21(17)$ & $3(4)$ & $23(28)$ \\
Panel GP & $28(19)$ & $13(19)$ & $9(12)$ & $19(24)$ \\
Surgeons & $28(32)$ & $12(10)$ & $10(8)$ & $18(24)$ \\
GPs & $21(22)$ & $13(11)$ & $16(17)$ & $5(5)$ \\
\hline
\end{tabular}

\section{Results}

After evaluating the clinical information only, the two consultant surgeons on the study panel each judged 3 admissions (6\%) as clearly inappropriate, the GP member judged 9, the larger group of surgeons $10(20 \%)$, and the group of GPs judged 16 admissions inappropriate (see table 1). All observers except consultant 2 judged the necessity of admission unclear in 12-14 cases (24-28\%). The addition of information on the home circumstances of the patient and the day and time of presentation to the $A \& E$ department had little effect except in the case of the GP member of the study panel. After studying this information, the consultant surgeons increased their evaluations of the number of inappropriate admissions to 4 and 6 respectively, while the GP on the panel increased his estimate to 12 , as well as increasing the number judged uncertain to $19(38 \%)$. The group of surgeons reduced their estimate of unnecessary admissions to 8, while the GPs increased their total to 17 (table 1). The kappa values for comparison of the same observer or group before and after the additional information was studied, confirmed good to very good agreement ${ }^{4}$ (ie little change) between the two evaluations from the same observer, except in the case of the panel GP (table 2).

Agreement between the different observer groups was expressed as kappa scores for assessment on clinical grounds only (table 3 ) and on all information (table 4). On clinical grounds only, the agreement between consultant surgeons was moderate, as was that between the

Table 2 Agreement between evaluations made before and after background information by the same observer or group (kappa values)

\begin{tabular}{llllll}
\hline & Consultant 1 & Consultant 2 & Panel GP & Surgeons & GPs \\
\hline $\begin{array}{l}\text { Consultant 1 } \\
\text { Consultant 2 }\end{array}$ & 0.84 & 0.64 & & & \\
$\begin{array}{l}\text { Panel GP } \\
\text { Surgeons }\end{array}$ & & & 0.41 & 0.75 & \\
GPs & & & & & 0.88 \\
\hline
\end{tabular}

Table 3 Inter-rater agreement on clinical data (kappa values)

\begin{tabular}{llllll}
\hline & Consultant 1 & Consultant 2 & Panel GP & Surgeons & GPs \\
\hline Consultant 1 & & & & & \\
Consultant 2 & 0.59 & & & & \\
Panel GP & 0.56 & 0.56 & & & \\
Surgeons & 0.42 & 0.26 & 0.42 & 0.34 & \\
GPs & 0.08 & 0.23 & 0.31 & 0.34 \\
\hline
\end{tabular}

Table 4 Inter-rater agreement on clinical and additional data (kappa values)

\begin{tabular}{llllll}
\hline & Consultant 1 & Consultant 2 & Panel GP & Surgeons & GPs \\
\hline Consultant 1 & & & & & \\
Consultant 2 & 0.43 & 0.31 & 0.18 & & \\
Panel GP & 0.45 & 0.38 & 0.28 & & \\
Surgeons & 0.12 & 0.25 & 0.19 & 0.35 & \\
GPs & & &
\end{tabular}

Table 5 Inter-rater agreement on clinical and additional data after consensus discussion (kappa values)

\begin{tabular}{llll}
\hline & Consultant 1 & Consultant 2 & Panel GP \\
\hline Consultant 1 & & & \\
Consultant 2 & 0.84 & & \\
Panel GP & 0.69 & 0.62 & \\
\hline
\end{tabular}

GP member of the panel and the consultants, and that of the consultants and the other surgical staff was fair to moderate. ${ }^{4}$ Agreement between GPs and the hospital based groups was fair to poor. These figures reflect the scoring in table 1 , in which the GPs consistently scored more admissions as unnecessary than the hospital based assessors. Using all information, the agreements remained similar except those involving the GP panel member, whose agreement with the consultants became fair to poor.

After conferring, the three study group members were able to agree on assessment of 40 cases $(80 \%)$, giving kappa values of between 0.62 and 0.84 (good to very good agreement) between members (table 5). Their consensus view was that 28 cases definitely required admission, 8 were equivocal, and 4 were definitely unjustified: they failed to agree on 10 cases.

\section{Discussion}

The recent increase in the number of emergency admissions to hospital has caused widespread concern. ${ }^{1-5}$ The change has coincided with the most significant period of change in the organisation of the health care delivery system for 40 years, and the relationship between the two events is under considerable scrutiny. The change to a purchaser/provider system has provided some perverse incentives that may encourage emergency admissions, largely through inadequacies in the contracting mechanism. The Patients' Charter has raised expectations among the public without boosting the resources available to meet these. The increasing frequency of complaints and litigation against doctors is thought to have increased the practice of defensive medicine, and it has been suggested that these influences may have combined to lower the threshold for emergency admission. ${ }^{26}$

This study of medical opinion suggested that between 8 and $34 \%$ of current acute surgical admissions are thought unnecessary by other doctors directly involved in dealing with such cases, with a further $20-38 \%$ being of unclear value. The figures illustrate the lack of agreement between clinicians in this important area, but even on the most conservative estimate, they suggest that substantial reductions in emergency admission rates are possible.

Whether this potential improvement can be realised will depend firstly on a clear consensus on what constitutes a necessary admission, secondly on accurate definition of the main factors responsible for unnecessary admissions, and thirdly on the potential for development of measures to counteract these. While some organisational and attitudinal factors may be easily addressed, others may require major investment or substantial reorganisation. A pre- 
vious study of emergency medical admissions found a similar proportion of potentially avoidable admissions, but suggested that savings from a change of practice would be small. ${ }^{7}$

The relatively small combined influences of the time of day and the social background to the admission in this study suggest that these factors do not play a major role in determining emergency admission. The decisions reached in this study had no practical consequences, and it is possible that GP behaviour in practice would be very different from their opinions as stated. Further work may be required to verify the accuracy in practice of the opinions expressed.

The potential influences on admission decisions are clearly multifarious, and may not be immediately obvious even to those making them. The perceptions of the admitting doctors about why cases which fail to meet agreed criteria were in fact admitted would nonetheless be valuable in generating hypotheses. We have begun prospective studies of this type. The cases of patients who had been examined in the $A \& E$ department, but not admitted, were not considered in this study, and it could be argued that these should have been included. The practical difficulty of obtaining full details of the complaint and background for a large number of $A \& E$ attendees is daunting, in view of the much less complete record keeping associated with such attendances. In the present context we decided not to study these cases, since there has been no suggestion that many patients are being inappropriately refused admission.

The clinicians normally involved in the process of emergency surgical admission are GPs and surgical trainees and consultants, who refer and accept such patients. We compared the assessments of these groups with those of three doctors with a special interest in this project. We hoped to use the assessments of this three doctor panel as a substitute for general clinical opinion. We found that agreement between the different groups was not, in fact, particularly good, and that there was a consistent pattern to the disagreement. GPs were consistently the most sceptical about the need for hospital admission, and their agreement with consultant surgeons was notably poor. This was somewhat unexpected, since previous studies by surgeons, and the subjective beliefs of many surgeons, suggested that GPs might refer patients with difficult social or other problems to hospital even when the clinical problem did not merit admission. ${ }^{8}$ The previous study was conducted entirely from a surgical viewpoint, and the difference between its findings and ours may be due to this fact. Our study considered the responses of all of the different groups, including the GPs to the same set of real clinical cases. Coast et al found GPs to be consistently less likely than hospital consultants to recommend emergency hospital admission for medical and geriatric patients. Their suggestion that the GP may have a greater understanding of the suitability of available alternative modes of care seems an equally probable explanation for our similar findings for surgical admissions.
Further studies of emergency admissions will require a standard working definition of necessity, particularly if an attempt is to be made to estimate the justifiability of admissions using objective scoring systems such as the admissions evaluation protocol (AEP). ${ }^{10}$ The heterogeneity of the views found in this study suggests that a measure of relevant clinical opinion may be difficult to obtain. A consensus view based on discussion among experienced clinicians aware of the issues raised in the study may be the best obtainable approximation to a "median clinical view". We have shown that such discussion can result in good agreement between the observers. It is hoped that such consensus groups can agree guidelines on the need for admission in various circumstances, and to identify the "triggers" which cause experienced clinicians to feel that hospital admission is justified, even when serious illness is not definitely present.

\section{Appendix I}

The following are examples of case studies prepared by the research nurse.

\section{CASE 1-A 73 YEAR OLD WOMAN}

Presentation

Several years' history of indigestion, heartburn, and epigastric pain.

Over the last two weeks, indigestion significantly worse and associated with colicky, lower abdominal pain. No radiation of pain.

Pain exacerbated by moving, but also occurs at rest.

For the last two days, patient vomited $(\times 3)$. No blood/coffee grounds.

No bowel or urinary symptoms.

Non-smoker.

Past history

As above. Nil else.

\section{Examination}

Looks comfortable; not distressed.

Temperature $35.0^{\circ} \mathrm{C}$; blood pressure 120 / $50 \mathrm{mmHg}$; pulse 70 (regular); respiration 18 .

Obese. ? Jaundiced sclera. Slightly dehydrated. Chest clear.

JVP >. Heart sounds normal. No oedema.

Abdominal examination: general tenderness, guarding and rebound, but soft-no rigidity.

Per rectum examination-not done.

\section{Investigations}

White cell count 8.6; haemoglobin 12.2; platelets 316 ; mean cell volume 92.7 .

$\mathrm{Na}+141 ; \mathrm{K}+3.4$; urea 10.0 ; creatinine 91 ; glucose 6.6; amylase 52 .

Chest $x$ ray-no free gas. Nil acute.

Abdominal $x$ ray-gas in descending colon; ascending colon more dilated than the rest of the colon; absence of gas at right end of transverse colon.

If it were your decision alone, would you have admitted this patient? 
- A. Definitely necessary on medical grounds;

- B. Unclear of necessity;

- C. Definitely not necessary on medical grounds.

\section{Social history}

Married, retired. Lives with husband in house with all amenities.

Generally fairly fit and well. Husband has coronary artery disease and needs assistance at all times with the activities of daily living.

Patient independent and self caring.

Bathroom and toilet upstairs, no chair lift in situ.

Two daughters help out with shopping, housework etc when needed.

Patient has home telephone. No private transport.

No social services input.

Time of admission

13.41

Day of admission

Wednesday

If it were your decision alone, would you still have admitted this patient?

- A. Definitely necessary on medical and non-medical grounds;

- B. Unclear of necessity;

- C. Definitely not necessary on medical and non-medical grounds.

\section{CASE HISTORY - 24 YEAR OLD MAN \\ Presentation}

Six hour history of abdominal pain. Pain in the abdominal region; intermittent and colicky.

No exacerbating or relieving factors.

Bowels last opened two days ago-normal stool, but blood on toilet pan.

Also complaining of increased abdominal distension.

No nausea or vomiting. No weight loss.

Non-smoker.

\section{Past history}

Laparotomy as a child for retrieval of razor blades (patient swallowed them).

Circumcision as a child.

Episode of bowel obstruction seven years ago.

\section{Examination}

Looks well.

Temperature $36.9^{\circ} \mathrm{C}$; blood pressure $138 /$

$76 \mathrm{mmHg}$; pulse 82 (regular).

Heart sounds normal. Nil added.

Chest clear.

Abdominal examination-soft, distended.

No tenderness, no guarding, no masses.

Bowel sounds + .
Per rectum examination-difficult, patient very tense.

No visible piles.

\section{Investigations}

White cell count 8.6 ; haemoglobin 14.7 ; platelets 251;

$\mathrm{Na}+137 ; \mathrm{K}+3.9$; urea 3.5 ; glucose 6.7 ; amylase 140 ;

Chest $x$ ray-not done.

Abdominal $x$ ray-some faecal loading.

If it were your decision alone, would you have admitted the patient?

- A. Definitely necessary on medical grounds;

- B. Unclear if necessary;

- C. Definitely not necessary on medical grounds.

\section{Social history}

Single, unemployed. Lives with grandmother in house with all amenities.

Patient independent and self caring.

Patient has a home telephone. No private transport.

Time of admission

01.34

Day of admission Sunday

If it were your decision alone would you still have admitted this patient?

- A. Definitely necessary on medical and non-medical grounds;

- B. Unclear if necessary;

- C. Definitely not necessary on medical and non-medical grounds.

We thank the staff of Fazakerley Hospital for their co-operation Professor Maggie Pearson for her helpful advice, and the general practitioners and surgeons involved for their participation.

Funding: this work was supported by a grant from the North West Regional Health Authority.

Conflict of interests: none.

1 Chappell AG. Patients have rising expectations. BMF 1995; 310:867-8.

2 Wardrope J, Kidner NL, Edhouse J. Bed crises are occurring almost daily in some hospitals. BMF 1995;310:868.

3 Audit Commission. Pan-Birmingham medical admissions study, phase 1. Birmingham, Audit Commission Regional Office, 1992 .

4 Brennan P, Silman A. Statistical measures for assessing observer variability in clinical measures. $B M \mathcal{F}$ 1992;304: $1491-4$.

5 Hobbs R. Rising emergency admissions. BMF 1995;310: 207-8.

6 Chadwick DR. GPs must take some responsibility. $B M F$ 1995;310:867.

7 Coast J, Inglis A, Morgan K, Gray S, et al. The hospital admissions study in England: are there alternatives to emergency hospital admission? $\mathcal{f}$ Epidemiol Community Health 1995;49:194-9.

8 Gaskell DJ, Lewis PA, Crosby DL, Roberts CJ, Fenn N, Roberts SM. Improving the primary management of emergency surgical admissions: a controlled trial. Ann $R$ Coll Surg Eng 1995;77:239-41.

9 Coast J, Inglis A, Frankel S. Alternatives to hospital care: what are they and who should decide? $B M \mathcal{F} 1996 ; 312$ :

10 Tsang $P$, Severs MP. A study of appropriateness of acute geriatric admissions and an assessment of the appropriateness evaluation protocol. $\mathcal{F}$ C Coll Physicians Lond 1995;29:311-4 